This is an electronic reprint of the original article. This reprint may differ from the original in pagination and typographic detail.

\author{
Author(s): Shi, Yue; Ward, D. E.; Carlsson, B. G.; Dobaczewski, Jacek; Nazarewicz, W.; Ragnarsson, \\ I.; Rudolph, D.
}

Title: $\quad$ Structure of superheavy nuclei along decay chains of element 115

Year: $\quad 2014$

Version:

Please cite the original version:

Shi, Y., Ward, D. E., Carlsson, B. G., Dobaczewski, J., Nazarewicz, W., Ragnarsson, I., \& Rudolph, D. (2014). Structure of superheavy nuclei along decay chains of element 115. Physical Review C, 90(1), Article 014308. https://doi.org/10.1103/PhysRevC.90.014308

All material supplied via JYX is protected by copyright and other intellectual property rights, and duplication or sale of all or part of any of the repository collections is not permitted, except that material may be duplicated by you for your research use or educational purposes in electronic or print form. You must obtain permission for any other use. Electronic or print copies may not be offered, whether for sale or otherwise to anyone who is not an authorised user. 


\title{
Structure of superheavy nuclei along decay chains of element 115
}

\author{
Yue Shi (石跃), ${ }^{1,2}$ D. E. Ward, ${ }^{3}$ B. G. Carlsson, ${ }^{3}$ J. Dobaczewski, ${ }^{4,5}$ W. Nazarewicz, ${ }^{1,4,6}$ I. Ragnarsson, ${ }^{3}$ and D. Rudolph ${ }^{7}$ \\ ${ }^{1}$ Department of Physics and Astronomy, University of Tennessee, Knoxville, Tennessee 37996, USA \\ ${ }^{2}$ Joint Institute for Heavy Ion Research, Oak Ridge National Laboratory, Oak Ridge, Tennessee 37831, USA \\ ${ }^{3}$ Division of Mathematical Physics, LTH, Lund University, SE-22100, Lund, Sweden \\ ${ }^{4}$ Institute of Theoretical Physics, Faculty of Physics, University of Warsaw, ul. Hoża 69, PL-00681 Warsaw, Poland \\ ${ }^{5}$ Department of Physics, Post Office Box 35 (YFL), FI-40014 University of Jyväskylä, Finland \\ ${ }^{6}$ Physics Division, Oak Ridge National Laboratory, Oak Ridge, Tennessee 37831, USA \\ ${ }^{7}$ Department of Physics, Lund University, SE-22100, Lund, Sweden
}

(Received 30 January 2014; revised manuscript received 10 June 2014; published 14 July 2014)

\begin{abstract}
A recent high-resolution $\alpha, X$-ray, and $\gamma$-ray coincidence-spectroscopy experiment offered the first glimpse of excitation schemes of isotopes along $\alpha$-decay chains of $Z=115$. To understand these observations and to make predictions about shell structure of superheavy nuclei below ${ }^{288} 115$, we employ two complementary mean-field models: the self-consistent Skyrme energy density functional approach and the macroscopic-microscopic Nilsson model. We discuss the spectroscopic information carried by the new data. In particular, candidates for the experimentally observed $E 1$ transitions in ${ }^{276} \mathrm{Mt}$ are proposed. We find that the presence and nature of low-energy $E 1$ transitions in well-deformed nuclei around $Z=110, N=168$ strongly depends on the strength of the spin-orbit coupling; hence, it provides an excellent constraint on theoretical models of superheavy nuclei. To clarify competing theoretical scenarios, an experimental search for $E 1$ transitions in odd- $A$ systems ${ }^{275,277} \mathrm{Mt}$, ${ }^{275} \mathrm{Hs}$, and ${ }^{277} \mathrm{Ds}$ is strongly recommended.
\end{abstract}

DOI: 10.1103/PhysRevC.90.014308

PACS number(s): 21.60.Jz, 23.20.Lv, 23.60.+e, 27.90.+b

\section{INTRODUCTION}

Superheavy nuclei at the limit of nuclear mass and atomic number pose a formidable challenge to both experiment and theory. The low cross sections for production of these nuclei, in the picobarn range or less, offer limited structural information. Moreover, the $\alpha$-decay chains of nuclei synthesized in experiments using a ${ }^{48} \mathrm{Ca}$ beam with actinide targets [1-9] terminate by spontaneous fission before reaching the known region of the nuclear chart. This poses a problem for the unambiguous identification of the new isotopes, and more direct techniques to determine $Z$ and $A$ must be employed [9]. Theoretical predictions of the shell structure of superheavy nuclei are also difficult, as the interplay between the electrostatic repulsion and nuclear attraction, combined with a very high density of single-particle (s.p.) states, make the results of calculations extremely sensitive to model details [10-15].

In a recent experimental study $[9,16]$, unique structural information on low-lying states in superheavy nuclei below ${ }^{288} 115$ has been obtained. Of particular interest is the finding that some of the measured transitions in the nucleus assigned to be ${ }^{276} \mathrm{Mt}$ have $E 1$ character, thus suggesting opposite parities of the connected states. The new data offer an exciting opportunity to constraint theoretical models in this region for the first time. Indeed, previous macroscopic-microscopic [17-19] and self-consistent studies [20,21] have shown that the number of opposite-parity s.p. orbitals around the Fermi level is fairly limited, and this is consistent with the Nilsson model analysis of Ref. [9].

Because of the above-mentioned sensitivity to model details, robust predictions in this region are difficult to make as one is dealing with large extrapolations. To this end, when aiming at reliable predictions, it is advisable to use a model that performs well in the neighboring region where experimental information is more abundant. Furthermore, since the quadrupole deformations of $\alpha$-decay daughters of ${ }^{288} 115$ are expected to increase gradually with decreasing $Z$ and $A$ along the $\alpha$-decay chain [13,14,17-21], shape polarization is going to play a role when determining the energies of low-lying states.

In this work, we study the low-lying states in the superheavy nuclei below ${ }^{288} 115$, using the locally optimized self-consistent Skyrme energy density functional (SEDF) and Nilsson-Strutinsky (NS) frameworks. To assess the robustness of these results, we also carry out calculations using a globally optimized SEDF model.

\section{MODELS}

The SEDF approach is a variant of nuclear density functional theory, which offers a global, self-consistent description of nuclear properties across the nuclear landscape [22,23]. The recent self-consistent study of Ref. [15] offers a locally optimized SEDF parametrization UNEDF1 ${ }^{\mathrm{SO}}$ that meets our local-extrapolability requirements: It reproduces one-quasiparticle (1-q.p.) states in ${ }^{251} \mathrm{Cf}$ and ${ }^{249} \mathrm{Bk}$ (the two heaviest systems where 1-q.p. energies are experimentally well known), predicts crucial deformed shell gaps at $N=152$ and $Z=100$, and describes rotational bands in Fm, No, and $\mathrm{Rf}$ isotopes. The parameter set $\mathrm{UNEDF} 1^{\mathrm{SO}}$ has been obtained by adjusting the spin-orbit coupling constants of a global SEDF parametrization UNEDF1 [24] that performs well for heavy nuclei and large deformations. We also use UNEDF1 in this study. The calculations closely follow Ref. [15]. The Skyrme Hartree-Fock-Bogolyubov (SHFB) equations were solved using the symmetry-unrestricted solver HFODD (v2.52j) [25] by expanding 1-q.p. wave functions in 680 deformed 


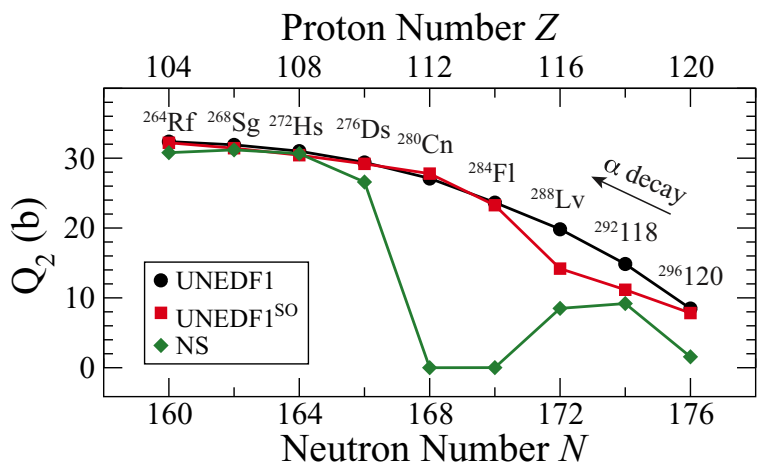

FIG. 1. (Color online) Quadrupole moments $Q_{2}$ of even-even nuclei forming the $\alpha$-decay chain ${ }^{296} 120 \rightarrow \cdots \rightarrow{ }^{264} \mathrm{Rf}$, calculated with UNEDF1 ${ }^{\text {SO }}$ and UNEDF1 SEDF models and the NS approach.

harmonic-oscillator (HO) basis states. To compute 1-q.p. excitations in odd- $A$ nuclei, we blocked relevant orbits around the Fermi level as described in Ref. [26]. The strengths of the pairing force for neutrons and protons were adjusted to the
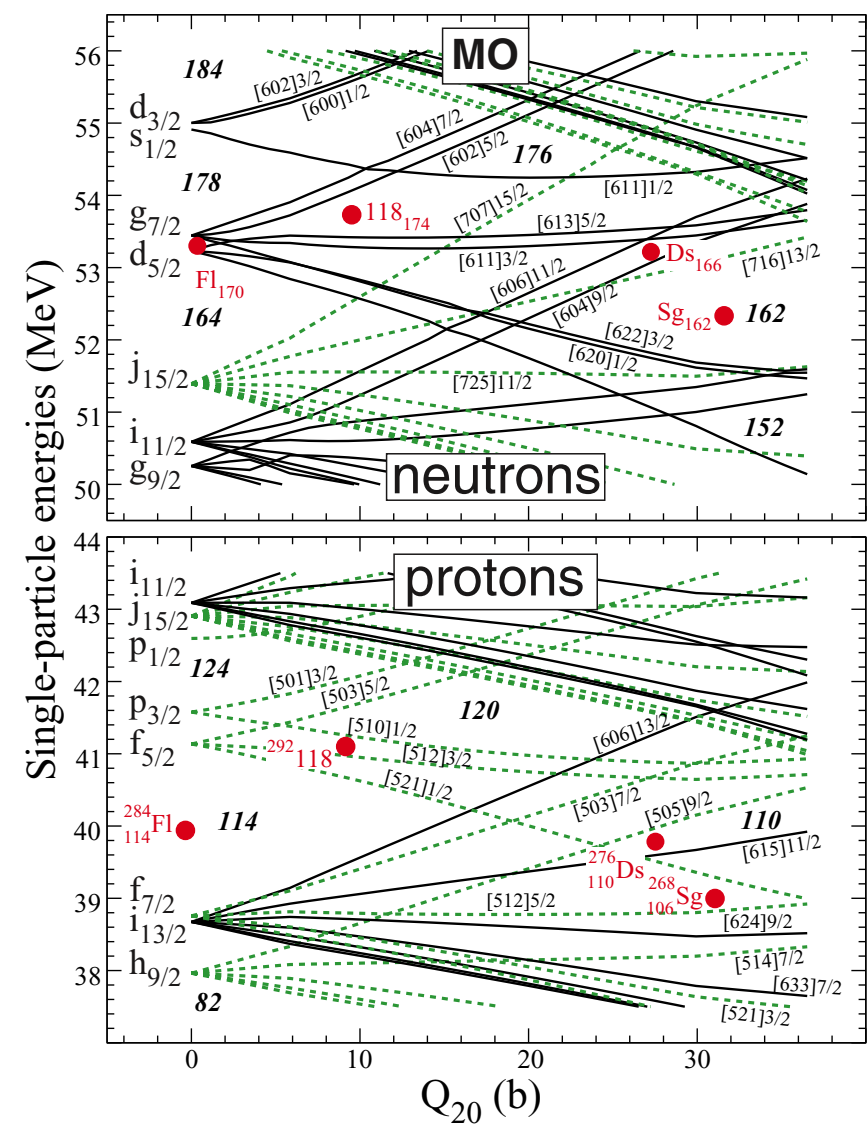

FIG. 2. (Color online) Nilsson diagram for neutrons (top) and protons (bottom) for nuclei along the $\alpha$-decay chain of ${ }^{296} 120$ using the MO potential of Ref. [29]. The orbits are labeled by the standard asymptotic Nilsson numbers [28]. The positive (negative) parity levels are marked by solid (dashed) lines. The Fermi levels of nuclei in Fig. 1 are indicated by dots. The quadrupole moment was determined from shape deformations $\epsilon_{2}$ and $\epsilon_{4}$ [34]: $Q_{20}=$ $0.8 A R_{0}^{2}\left(\epsilon_{2}+0.5 \epsilon_{2}^{2}+0.758 \epsilon_{4}^{2}-\epsilon_{2} \epsilon_{4}\right)$ with $A=280, R_{0}=r_{0} A^{1 / 3}$, and $r_{0}=1.217 \mathrm{fm}$.

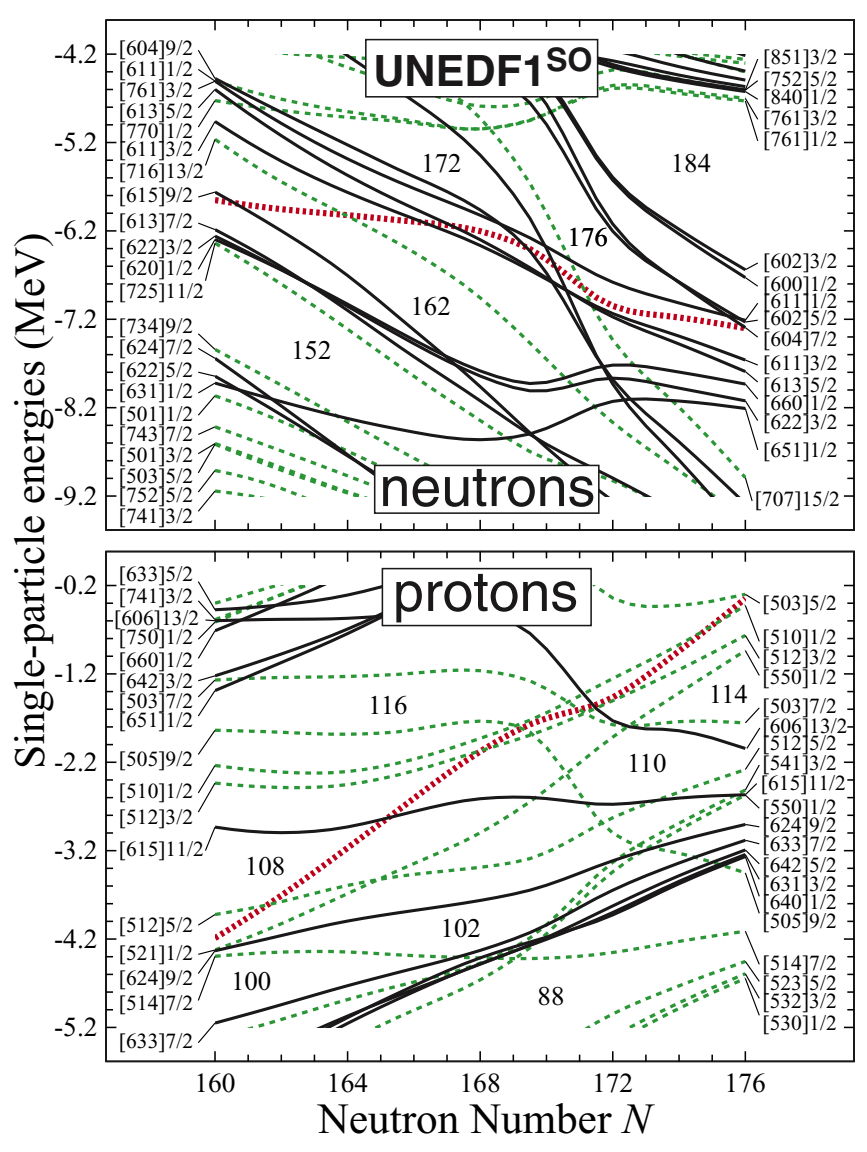

FIG. 3. (Color online) Single-neutron (top) and single-proton (bottom) canonical energies of $\mathrm{UNEDF}^{\mathrm{SO}}$ for nuclei along the $\alpha$ decay chain of ${ }^{296} 120$ as in Fig. 1. The orbits are labeled by the standard asymptotic Nilsson numbers corresponding to the dominant components of the SHFB canonical wave functions. The positive (negative) parity levels are marked by solid (dashed) lines. The Fermi levels are indicated by thick dotted lines.

odd-even mass staggering in ${ }^{251} \mathrm{Cf}$ and ${ }^{249} \mathrm{Bk}$ and the kinematic moment of inertia of ${ }^{252}$ No.

The SEDF results are compared with those of the NilssonStrutinsky (NS) approach of Ref. [27] with the modified harmonic oscillator (MO) potential and pairing as in Ref. [28]. The shell-independent $\mathrm{MO}$ parameters $\left(\kappa_{p}=0.058, \mu_{p}=0.63\right.$, $\kappa_{n}=0.0526$, and $\mu_{n}=0.457$ ) have been locally optimized to the actinide nuclei [29] and applied to, e.g., ${ }^{228,230} \mathrm{~Pa}[30]$ and ${ }^{242} \mathrm{Am}[31]$.

The 2-q.p.-plus-rotor calculations for odd-odd nuclei were carried out using the MO model of Ref. [32]. The moments of inertia were chosen according to a phenomenological relation of Ref. [33]. The BCS pairing was treated as in Ref. [28], with the monopole pairing strengths taken as $95 \%$ of the values for even-even nuclei. No residual proton-neutron interaction was considered.

\section{RESULTS}

We first discuss properties of the even-even nuclei belonging to the $\alpha$-decay chain of ${ }^{296} 120$. Their ground states form 


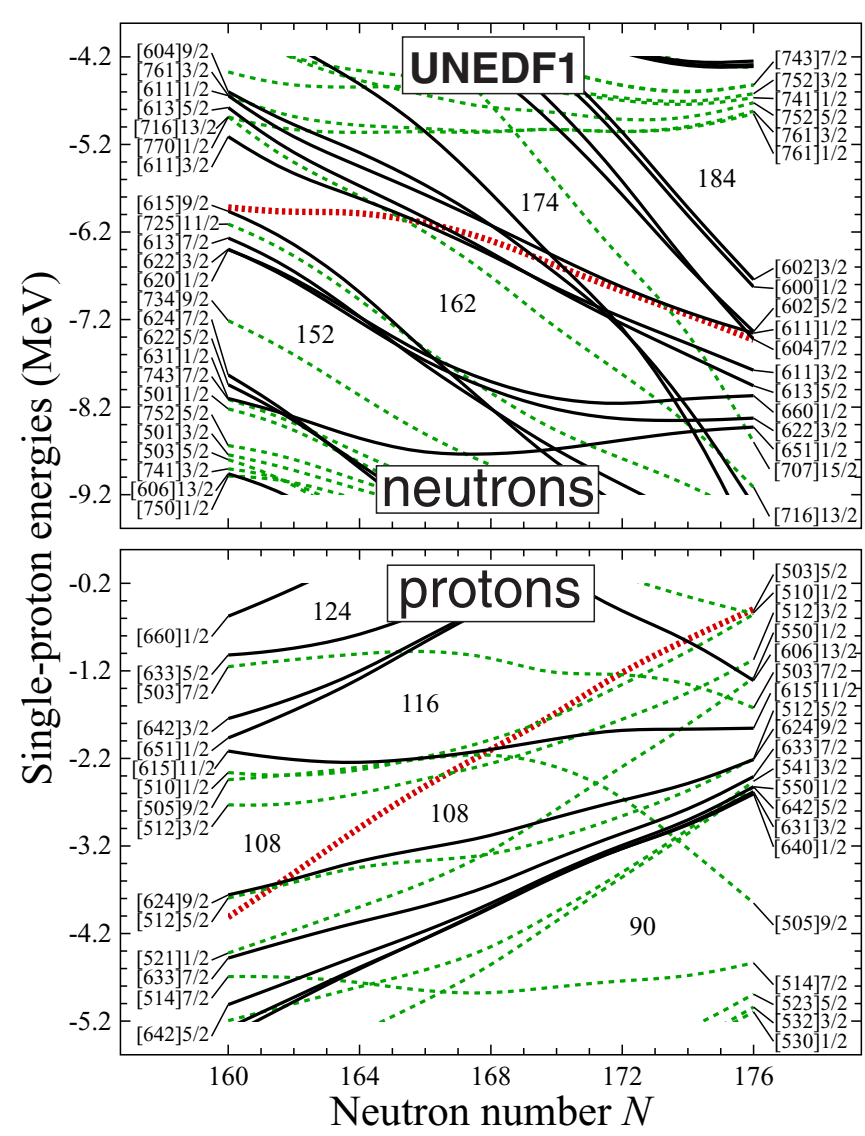

FIG. 4. (Color online) Similar as in Fig. 3 but for UNEDF1.

q.p. vacua for neighboring odd- $A$ and odd-odd systems. The calculated quadrupole moments are shown in Fig. 1. Both SEDF models predict a similar smooth increase of quadrupole deformation along the $\alpha$ chain. In the NS calculations, ${ }^{296} 120$ is nearly spherical, ${ }^{292} 118$ and ${ }^{288} \mathrm{Lv}$ are very weakly deformed, ${ }^{284} \mathrm{Fl}$ and ${ }^{280} \mathrm{Cn}$ are spherical, and the shapes of the lightest daughters have deformations close to those predicted by SEDF. These results suggest that a direct comparison between SEDF and NS models is most meaningful for $Z<112$.

It is instructive to begin the discussion from the Nilsson s.p. diagram of the MO potential shown in Fig. 2. The main features of this diagram, such as the appearance of spherical shell gaps at $Z=114$ and $N=184$, have remained unchanged since the late 1960s [35,36]. It is worth noting that the s.p. spectrum of the MO model, with its pronounced spherical shell gaps at $Z=114$ and $N=184$ and resulting Nilsson orbits, is fairly close to that of more realistic Woods-Saxon [17-19] and folded-Yukawa [37-39] potentials; see Refs. [10,40] for more discussion.

The deformed shell structure of nuclei at the end of the $\alpha$-decay chain of ${ }^{296} 120$ (or ${ }^{288} 115$ ) is relatively simple: Both in neutrons and protons there appears one unique-parity, high- $\Omega$ Nilsson state $(v[716] 13 / 2$ and $\pi[615] 11 / 2)$ surrounded by levels of opposite parity, such as neutron ([613]5/2, [611]3/2), ([606]11/2, [604]9/2) and proton ([503]7/2, [505]9/2), ([510]1/2, [512]3/2) pseudospin doublets, respectively.
TABLE I. Excitation energies, total quadrupole moments, and quadrupole mass deformations $\beta_{2}$ for one-quasiparticle excitations in selected nuclei belonging to the $\alpha$-decay chains of ${ }^{287,289} \mathrm{Lv}$ predicted with $\mathrm{UNEDF}^{\mathrm{SO}}{ }^{\mathrm{SO}}$. The high- $\Omega$ unique-parity states are printed in boldface. The intrinsic configurations are labeled as in Fig. 3. The predictions on the lightest member of ${ }^{289} \mathrm{Lv} \alpha$-decay chain, ${ }^{277} \mathrm{Ds}$, are discussed below.

\begin{tabular}{|c|c|c|c|c|}
\hline Nucleus & Config. & $E_{x}(\mathrm{MeV})$ & $Q_{20}$ (b) & $\beta_{2}$ \\
\hline \multirow{5}{*}{${ }_{110}^{275} \mathrm{Ds}_{165}$} & {$[611] 3 / 2$} & 0 & 29.8 & 0.23 \\
\hline & {$[613] 5 / 2$} & 0.085 & 29.7 & 0.23 \\
\hline & {$[716] 13 / 2$} & 0.151 & 29.9 & 0.23 \\
\hline & {$[611] 1 / 2$} & 0.305 & 29.8 & 0.23 \\
\hline & {$[604] 9 / 2$} & 0.619 & 29.4 & 0.22 \\
\hline \multirow[t]{5}{*}{${ }_{112}^{279} \mathrm{Cn}_{167}$} & {$[611] 3 / 2$} & 0 & 28.3 & 0.21 \\
\hline & {$[613] 5 / 2$} & 0.013 & 28.3 & 0.21 \\
\hline & {$[611] 1 / 2$} & 0.121 & 28.3 & 0.21 \\
\hline & {$[604] 9 / 2$} & 0.306 & 28.0 & 0.21 \\
\hline & {$[716] 13 / 2$} & 0.627 & 28.5 & 0.22 \\
\hline \multirow[t]{5}{*}{${ }_{112}^{281} \mathrm{Cn}_{169}$} & {$[611] 1 / 2$} & 0 & 27.2 & 0.20 \\
\hline & {$[604] 9 / 2$} & 0.145 & 27.4 & 0.21 \\
\hline & {$[613] 5 / 2$} & 0.159 & 27.1 & 0.20 \\
\hline & {$[611] 3 / 2$} & 0.237 & 26.2 & 0.20 \\
\hline & {$[606] 11 / 2$} & 0.606 & 24.8 & 0.19 \\
\hline \multirow[t]{5}{*}{${ }_{114}^{283} \mathrm{Fl}_{169}$} & {$[611] 1 / 2$} & 0 & 25.4 & 0.19 \\
\hline & {$[604] 9 / 2$} & 0.165 & 25.0 & 0.19 \\
\hline & {$[613] 5 / 2$} & 0.184 & 24.9 & 0.19 \\
\hline & {$[611] 3 / 2$} & 0.208 & 24.5 & 0.18 \\
\hline & {$[606] 11 / 2$} & 0.399 & 23.6 & 0.18 \\
\hline \multirow[t]{5}{*}{${ }_{114}^{285} \mathrm{Fl}_{171}$} & {$[611] 1 / 2$} & 0 & 21.0 & 0.15 \\
\hline & {$[613] 5 / 2$} & 0.051 & 19.0 & 0.14 \\
\hline & {$[611] 3 / 2$} & 0.056 & 19.0 & 0.14 \\
\hline & {$[606] 11 / 2$} & 0.058 & 21.0 & 0.15 \\
\hline & {$[707] 15 / 2$} & 0.232 & 19.6 & 0.14 \\
\hline \multirow[t]{6}{*}{${ }_{116}^{287} \mathrm{Lv}_{171}$} & {$[613] 5 / 2$} & 0 & 14.2 & 0.10 \\
\hline & {$[611] 3 / 2$} & 0.085 & 14.2 & 0.10 \\
\hline & {$[707] 15 / 2$} & 0.219 & 14.8 & 0.10 \\
\hline & {$[611] 1 / 2$} & 0.314 & 14.3 & 0.10 \\
\hline & {$[622] 3 / 2$} & 0.378 & 13.1 & 0.09 \\
\hline & {$[604] 9 / 2$} & 0.495 & 16.2 & 0.12 \\
\hline \multirow[t]{5}{*}{${ }_{116}^{289} \mathrm{Lv}_{173}$} & {$[613] 5 / 2$} & 0 & 11.8 & 0.08 \\
\hline & {$[611] 3 / 2$} & 0.029 & 11.8 & 0.08 \\
\hline & {$[611] 1 / 2$} & 0.055 & 11.9 & 0.08 \\
\hline & {$[604] 7 / 2$} & 0.372 & 11.2 & 0.08 \\
\hline & {$[602] 5 / 2$} & 0.397 & 11.3 & 0.08 \\
\hline
\end{tabular}

The spherical shell structure in superheavy nuclei strongly depends on the spin-orbit splitting, which governs the size of the $Z=114$ gap (cf. Table IV of Ref. [10] and discussion therein). Also, the coupling between Coulomb interaction and nuclear interaction is expected to impact the predictions. To consider both effects, we studied s.p. canonical states obtained with UNEDF1 ${ }^{\text {SO }}$ and UNEDF1 SEDF models, which differ in the spin-orbit sector and treat the electrostatic energy selfconsistently.

The s.p. energies of $\mathrm{UNEDF}^{\mathrm{SO}}$ along the $\alpha$-decay chain of ${ }^{296} 120$ are depicted in Fig. 3. 
TABLE II. Similar as in Table I but for UNEDF1.

\begin{tabular}{|c|c|c|c|c|}
\hline Nucleus & Config. & $E_{x}(\mathrm{MeV})$ & $Q_{20}(\mathrm{~b})$ & $\beta_{2}$ \\
\hline \multirow{5}{*}{${ }_{110}^{275} \mathrm{Ds}_{165}$} & {$[613] 5 / 2$} & 0 & 30.2 & 0.23 \\
\hline & {$[611] 3 / 2$} & 0.050 & 30.2 & 0.23 \\
\hline & {$[716] 13 / 2$} & 0.121 & 30.2 & 0.23 \\
\hline & {$[611] 1 / 2$} & 0.364 & 30.2 & 0.23 \\
\hline & {$[604] 9 / 2$} & 0.561 & 29.8 & 0.23 \\
\hline \multirow[t]{5}{*}{${ }_{112}^{279} \mathrm{Cn}_{167}$} & {$[611] 3 / 2$} & 0 & 28.1 & 0.21 \\
\hline & {$[613] 5 / 2$} & 0.044 & 28.1 & 0.21 \\
\hline & {$[611] 1 / 2$} & 0.074 & 28.2 & 0.21 \\
\hline & {$[716] 13 / 2$} & 0.197 & 28.3 & 0.21 \\
\hline & {$[604] 9 / 2$} & 0.201 & 27.8 & 0.21 \\
\hline \multirow[t]{5}{*}{${ }_{112}^{281} \mathrm{Cn}_{169}$} & {$[611] 1 / 2$} & 0 & 26.3 & 0.19 \\
\hline & {$[604] 9 / 2$} & 0.109 & 26.2 & 0.19 \\
\hline & {$[611] 3 / 2$} & 0.152 & 25.7 & 0.19 \\
\hline & {$[613] 5 / 2$} & 0.157 & 26.0 & 0.19 \\
\hline & {$[606] 11 / 2$} & 0.267 & 25.0 & 0.19 \\
\hline \multirow[t]{5}{*}{${ }_{114}^{283} \mathrm{Fl}_{169}$} & {$[611] 1 / 2$} & 0 & 25.5 & 0.19 \\
\hline & {$[604] 9 / 2$} & 0.114 & 25.1 & 0.19 \\
\hline & {$[611] 3 / 2$} & 0.147 & 24.7 & 0.18 \\
\hline & {$[613] 5 / 2$} & 0.160 & 24.6 & 0.18 \\
\hline & {$[606] 11 / 2$} & 0.195 & 24.3 & 0.18 \\
\hline \multirow[t]{6}{*}{${ }_{114}^{285} \mathrm{Fl}_{171}$} & {$[604] 9 / 2$} & 0 & 22.8 & 0.17 \\
\hline & {$[611] 1 / 2$} & 0.009 & 22.2 & 0.17 \\
\hline & {$[611] 3 / 2$} & 0.139 & 21.5 & 0.16 \\
\hline & {$[606] 11 / 2$} & 0.169 & 22.2 & 0.17 \\
\hline & {$[613] 5 / 2$} & 0.184 & 21.4 & 0.16 \\
\hline & {$[707] 15 / 2$} & 0.723 & 20.4 & 0.15 \\
\hline \multirow{6}{*}{${ }_{116}^{287} \mathrm{Lv}_{171}$} & {$[604] 9 / 2$} & 0 & 21.8 & 0.16 \\
\hline & {$[611] 1 / 2$} & 0.003 & 21.2 & 0.15 \\
\hline & {$[611] 3 / 2$} & 0.113 & 20.5 & 0.15 \\
\hline & {$[613] 5 / 2$} & 0.175 & 20.4 & 0.15 \\
\hline & {$[606] 11 / 2$} & 0.204 & 21.2 & 0.15 \\
\hline & {$[707] 15 / 2$} & 0.661 & 19.2 & 0.14 \\
\hline \multirow[t]{5}{*}{${ }_{116}^{289} \mathrm{Lv}_{173}$} & {$[611] 1 / 2$} & 0 & 18.1 & 0.13 \\
\hline & {$[611] 3 / 2$} & 0.256 & 17.7 & 0.13 \\
\hline & {$[613] 5 / 2$} & 0.394 & 17.8 & 0.13 \\
\hline & {$[604] 9 / 2$} & 0.649 & 18.4 & 0.13 \\
\hline & {$[707] 15 / 2$} & 0.796 & 18.0 & 0.13 \\
\hline
\end{tabular}

The s.p. neutron spectrum is dominated by deformed gaps at $N=152$ and 162, and a large spherical shell gap at $N=184$. In the deformed region $160 \leqslant N \leqslant 168$, the Nilsson states close to the Fermi level are primarily $N_{\text {osc }}=6$ levels and one unique-parity, high- $\Omega$ intruder level $\nu[716] 13 / 2$ originating from the spherical $1 j_{15 / 2}$ shell. The structure of the proton Nilsson diagram in Fig. 3 is dominated by deformed gaps at $Z=100,102$, and 108, and a spherical subshell closure at $Z=$ 114. The unique-parity, high- $\Omega$ intruder level $\pi[615] 11 / 2$ originating from the spherical $1 i_{13 / 2}$ shell is surrounded by several $N_{\text {osc }}=5$ Nilsson orbitals.

The general pattern of s.p. states predicted by $\mathrm{UNEDF}^{\mathrm{SO}}$ is not that far from that in Fig. 2 of the MO potential. However, there are differences in the spherical shell structure, which will impact detailed predictions for deformed superheavy nuclei belonging to $Z=115 \alpha$-decay chains. In particular,
TABLE III. Similar as in Table I but for one-quasiproton excitations in the $\alpha$-decay chains of ${ }^{293} 117$ and ${ }^{287} 115$ predicted with $\mathrm{UNEDF} 1^{\mathrm{SO}}$.

\begin{tabular}{|c|c|c|c|c|}
\hline Nucleus & Config. & $E_{x}(\mathrm{MeV})$ & $Q_{20}(\mathrm{~b})$ & $\beta_{2}$ \\
\hline \multirow{5}{*}{${ }_{111}^{279} \operatorname{Rg}_{168}$} & {$[512] 3 / 2$} & 0 & 28.3 & 0.21 \\
\hline & {$[510] 1 / 2$} & 0.122 & 28.3 & 0.21 \\
\hline & {$[615] 11 / 2$} & 0.284 & 28.4 & 0.21 \\
\hline & {$[505] 9 / 2$} & 0.392 & 27.7 & 0.20 \\
\hline & {$[521] 1 / 2$} & 0.633 & 26.6 & 0.19 \\
\hline \multirow{5}{*}{${ }_{111}^{281} \operatorname{Rg}_{170}$} & {$[512] 3 / 2$} & 0 & 26.0 & 0.20 \\
\hline & {$[510] 1 / 2$} & 0.165 & 26.1 & 0.20 \\
\hline & {$[505] 9 / 2$} & 0.263 & 25.8 & 0.20 \\
\hline & {$[615] 11 / 2$} & 0.277 & 26.1 & 0.20 \\
\hline & {$[521] 1 / 2$} & 0.412 & 25.3 & 0.19 \\
\hline \multirow{5}{*}{${ }^{283} 113_{170}$} & {$[512] 3 / 2$} & 0 & 24.5 & 0.18 \\
\hline & {$[510] 1 / 2$} & 0.057 & 24.5 & 0.18 \\
\hline & {$[505] 9 / 2$} & 0.090 & 24.4 & 0.18 \\
\hline & {$[503] 7 / 2$} & 0.453 & 22.9 & 0.16 \\
\hline & {$[521] 1 / 2$} & 0.629 & 23.2 & 0.16 \\
\hline \multirow[t]{6}{*}{${ }^{285} 113_{172}$} & {$[512] 3 / 2$} & 0 & 18.7 & 0.14 \\
\hline & {$[503] 7 / 2$} & 0.096 & 17.6 & 0.13 \\
\hline & {$[550] 1 / 2$} & 0.146 & 17.2 & 0.13 \\
\hline & {$[510] 1 / 2$} & 0.177 & 19.4 & 0.15 \\
\hline & {$[505] 9 / 2$} & 0.380 & 19.7 & 0.15 \\
\hline & {$[615] 11 / 2$} & 0.645 & 18.2 & 0.14 \\
\hline \multirow[t]{5}{*}{${ }^{287} 115_{172}$} & {$[512] 3 / 2$} & 0 & 14.4 & 0.10 \\
\hline & {$[550] 1 / 2$} & 0.042 & 12.6 & 0.09 \\
\hline & {$[503] 7 / 2$} & 0.127 & 14.2 & 0.10 \\
\hline & {$[606] 13 / 2$} & 0.206 & 14.2 & 0.10 \\
\hline & {$[510] 1 / 2$} & 0.388 & 14.1 & 0.10 \\
\hline \multirow[t]{5}{*}{${ }^{289} 115_{174}$} & {$[550] 1 / 2$} & 0 & 11.3 & 0.08 \\
\hline & {$[512] 3 / 2$} & 0.002 & 11.5 & 0.08 \\
\hline & {$[606] 13 / 2$} & 0.137 & 12.2 & 0.09 \\
\hline & {$[503] 7 / 2$} & 0.230 & 11.6 & 0.08 \\
\hline & {$[510] 1 / 2$} & 0.338 & 11.6 & 0.08 \\
\hline \multirow[t]{5}{*}{${ }^{293} 117_{176}$} & {$[512] 3 / 2$} & 0 & 7.8 & 0.05 \\
\hline & {$[550] 1 / 2$} & 0.06 & 7.5 & 0.05 \\
\hline & {$[510] 1 / 2$} & 0.218 & 7.8 & 0.05 \\
\hline & {$[503] 5 / 2$} & 0.310 & 7.0 & 0.04 \\
\hline & {$[503] 7 / 2$} & 0.778 & 8.1 & 0.06 \\
\hline
\end{tabular}

MO predicts larger spherical shell gaps at $Z=114, N=148$, and $N=178$. In $\mathrm{UNEDF}^{\mathrm{SO}}$, the splitting between the $1 j_{15 / 2}$ and $1 i_{11 / 2}$ spherical neutron shells is very small. This results in an upward shift of the ([606]11/2, [604]9/2) doublet.

As seen in Fig. 4, in the case of UNEDF1 the unique-parity $\nu 1 j_{15 / 2}$ and $\pi 1 i_{13 / 2}$ shells are shifted up by a few hundred $\mathrm{keV}$, which results in a significant reduction of spherical $N=164$ and $Z=114$ shell closures [15]. The change in the spin-orbit potential also impacts positions of deformed levels. In particular, the deformed neutron gap at $N=152$ is reduced, and that at $N=162$ opens up. In the proton sector, the deformed Nilsson state [615]11/2 appears just below the significantly increased $Z=116$ gap, close to the [505]9/2 and [510]1/2 levels. The second proton intruder state [624]9/2 shows up just below the deformed proton gap at $Z=108$. 
TABLE IV. Similar as in Table III but with UNEDF1.

\begin{tabular}{|c|c|c|c|c|}
\hline Nucleus & Config. & $E_{x}(\mathrm{MeV})$ & $Q_{20}(\mathrm{~b})$ & $\beta_{2}$ \\
\hline \multirow{5}{*}{${ }_{111}^{279} \operatorname{Rg}_{168}$} & {$[512] 3 / 2$} & 0 & 27.8 & 0.21 \\
\hline & {$[615] 11 / 2$} & 0.075 & 27.8 & 0.21 \\
\hline & {$[505] 9 / 2$} & 0.119 & 27.6 & 0.21 \\
\hline & {$[510] 1 / 2$} & 0.252 & 27.8 & 0.21 \\
\hline & {$[624] 9 / 2$} & 0.793 & 27.6 & 0.21 \\
\hline \multirow[t]{5}{*}{${ }_{111}^{281} \operatorname{Rg}_{170}$} & {$[615] 11 / 2$} & 0 & 25.3 & 0.19 \\
\hline & {$[512] 3 / 2$} & 0.021 & 25.3 & 0.19 \\
\hline & {$[505] 9 / 2$} & 0.087 & 25.4 & 0.19 \\
\hline & {$[510] 1 / 2$} & 0.218 & 25.3 & 0.19 \\
\hline & {$[521] 1 / 2$} & 0.601 & 23.9 & 0.18 \\
\hline \multirow{5}{*}{${ }^{283} 113_{170}$} & {$[510] 1 / 2$} & 0 & 24.3 & 0.18 \\
\hline & {$[512] 3 / 2$} & 0.024 & 24.1 & 0.18 \\
\hline & {$[615] 11 / 2$} & 0.055 & 24.3 & 0.18 \\
\hline & {$[503] 7 / 2$} & 0.480 & 22.7 & 0.17 \\
\hline & {$[550] 1 / 2$} & 0.702 & 22.7 & 0.17 \\
\hline \multirow[t]{5}{*}{${ }^{285} 113_{172}$} & {$[510] 1 / 2$} & 0 & 21.2 & 0.16 \\
\hline & {$[512] 3 / 2$} & 0.044 & 21.0 & 0.15 \\
\hline & {$[615] 11 / 2$} & 0.089 & 21.1 & 0.15 \\
\hline & {$[505] 9 / 2$} & 0.287 & 22.8 & 0.16 \\
\hline & {$[550] 1 / 2$} & 0.411 & 19.8 & 0.15 \\
\hline \multirow[t]{5}{*}{${ }^{287} 115_{172}$} & {$[510] 1 / 2$} & 0 & 20.2 & 0.15 \\
\hline & {$[503] 7 / 2$} & 0.055 & 20.1 & 0.15 \\
\hline & {$[512] 3 / 2$} & 0.193 & 20.1 & 0.15 \\
\hline & {$[615] 11 / 2$} & 0.253 & 20.4 & 0.15 \\
\hline & {$[550] 1 / 2$} & 0.682 & 19.1 & 0.14 \\
\hline \multirow[t]{5}{*}{${ }^{289} 115_{174}$} & {$[503] 7 / 2$} & 0 & 17.1 & 0.12 \\
\hline & {$[512] 3 / 2$} & 0.024 & 16.9 & 0.12 \\
\hline & {$[510] 1 / 2$} & 0.085 & 17.1 & 0.12 \\
\hline & {$[615] 11 / 2$} & 0.272 & 17.3 & 0.12 \\
\hline & {$[550] 1 / 2$} & 0.438 & 16.5 & 0.12 \\
\hline \multirow[t]{7}{*}{${ }^{293} 117_{176}$} & {$[510] 1 / 2$} & 0 & 11.4 & 0.08 \\
\hline & {$[606] 13 / 2$} & 0.003 & 11.5 & 0.08 \\
\hline & {$[512] 3 / 2$} & 0.03 & 11.2 & 0.08 \\
\hline & {$[550] 1 / 2$} & 0.197 & 9.7 & 0.06 \\
\hline & {$[503] 5 / 2$} & 0.26 & 11.1 & 0.08 \\
\hline & {$[503] 7 / 2$} & 0.266 & 11.7 & 0.08 \\
\hline & {$[615] 11 / 2$} & 0.588 & 11.4 & 0.08 \\
\hline
\end{tabular}

\section{A. One-quasiparticle energies}

To get more insights, we computed the energies of 1-q.p. excitations for odd- $Z$, even- $N$ superheavy nuclei that form the $\alpha$-decay chains of ${ }_{116}^{287} \mathrm{Lv}_{171}$ and ${ }_{116}^{289} \mathrm{Lv}_{173}$ (Tables I and II), and ${ }^{287} 115_{172}$ and ${ }^{293} 117_{176}$ (Tables III and IV); the theoretical error on 1-q.p. excitations due to the adopted size of the HO basis is less than $60 \mathrm{keV}$ when going from 680 stretched $\mathrm{HO}$ states to 969 states. The results for ${ }_{116}^{287} \operatorname{Lv}_{171}$ and ${ }^{293} 117_{176}$ are shown in Figs. 5 and 6, respectively.

Although s.p. energies are not experimental observables, those around the Fermi level carry information about the lowlying q.p. configurations in neighboring odd- $A$ and odd-odd nuclei.

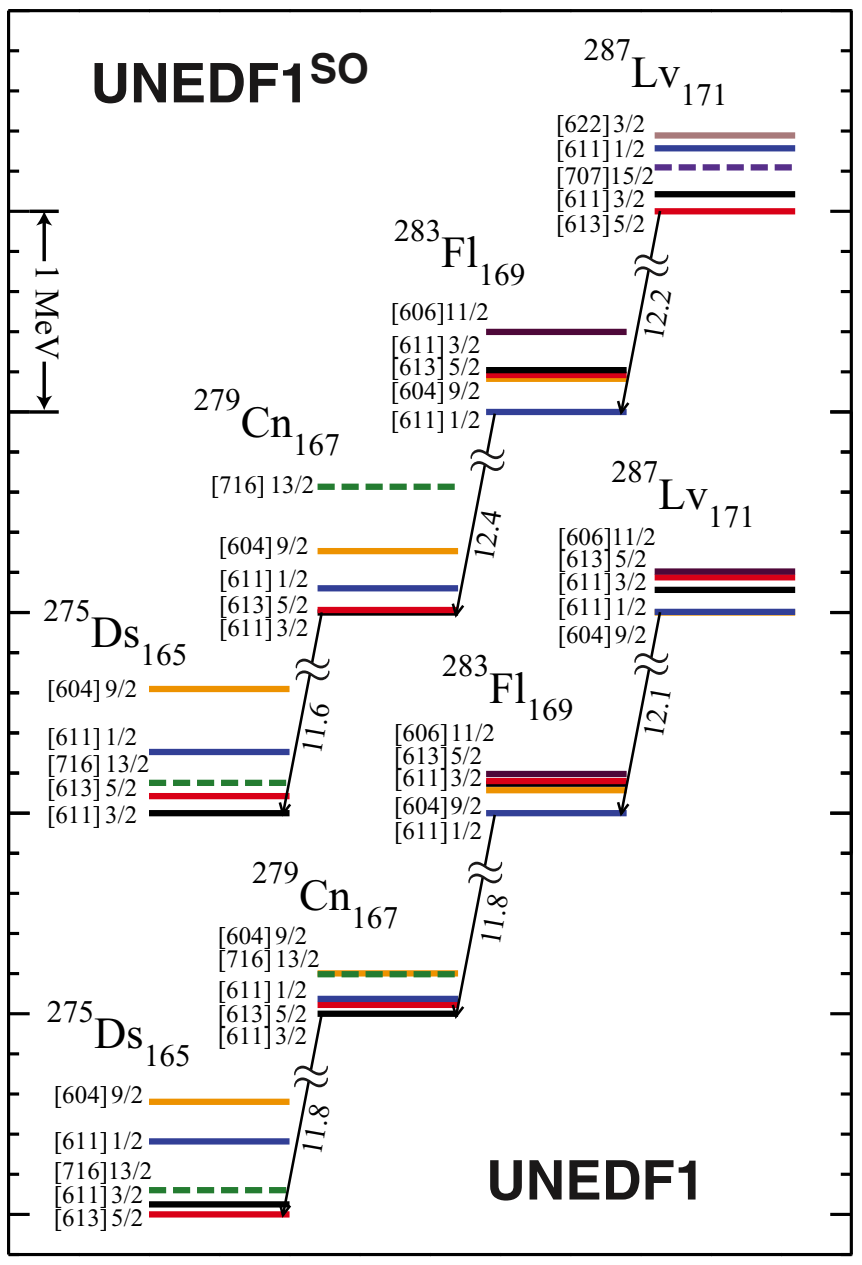

FIG. 5. (Color online) One-q.p. spectra for nuclei forming the $\alpha$-decay chain ${ }^{287} \mathrm{Lv} \rightarrow \cdots \rightarrow{ }^{275} \mathrm{Ds}$ predicted with $\mathrm{UNEDF}^{\mathrm{SO}}$ (upper sequence) and UNEDF1 (lower sequence). $Q_{\alpha}$ values for g.s. $\rightarrow$ g.s. transitions are marked. The binding energy differences between different nuclei are shifted arbitrarily, whereas the excitation energies within a given nucleus are shown to scale.

\section{B. Odd-odd nuclei}

By combining the low-lying 1-q.p. excitations, one can deduce possible 2-q.p. states in the odd-odd nuclei that form $\alpha$-decay chains of ${ }^{288} 115$. It is worth noting that there exist detailed calculations of 1-q.p. excitations in the heaviest elements using the macroscopic-microscopic Woods-Saxon model [17-19]; unfortunately, they cannot be used to assess the neutron s.p. structure in the region of interest as the range of neutron numbers covered $(N \leqslant 161)$ in these papers is too limited.

Let us look into the structure of ${ }^{276} \mathrm{Mt}$ in some detail. The structural information relevant to this nucleus is contained in the 1-q.p. spectra of its odd- $A$ neighbors ${ }^{275,277} \mathrm{Mt},{ }^{275} \mathrm{Hs}$, and ${ }^{277}$ Ds, provided in SEDF Table V. All low-lying 1-q.p. states in these nuclei correspond to very similar quadrupole mass deformation of $\beta_{2} \approx 0.22$, which facilitates comparison with the Nilsson diagram of Fig. 3. The lowest 1-q.p. proton states are the unique-parity [615]11/2 and $N_{\text {osc }}=5$ excitations [512]3/2, [521]1/2, [510]1/2, and [512]5/2. The 


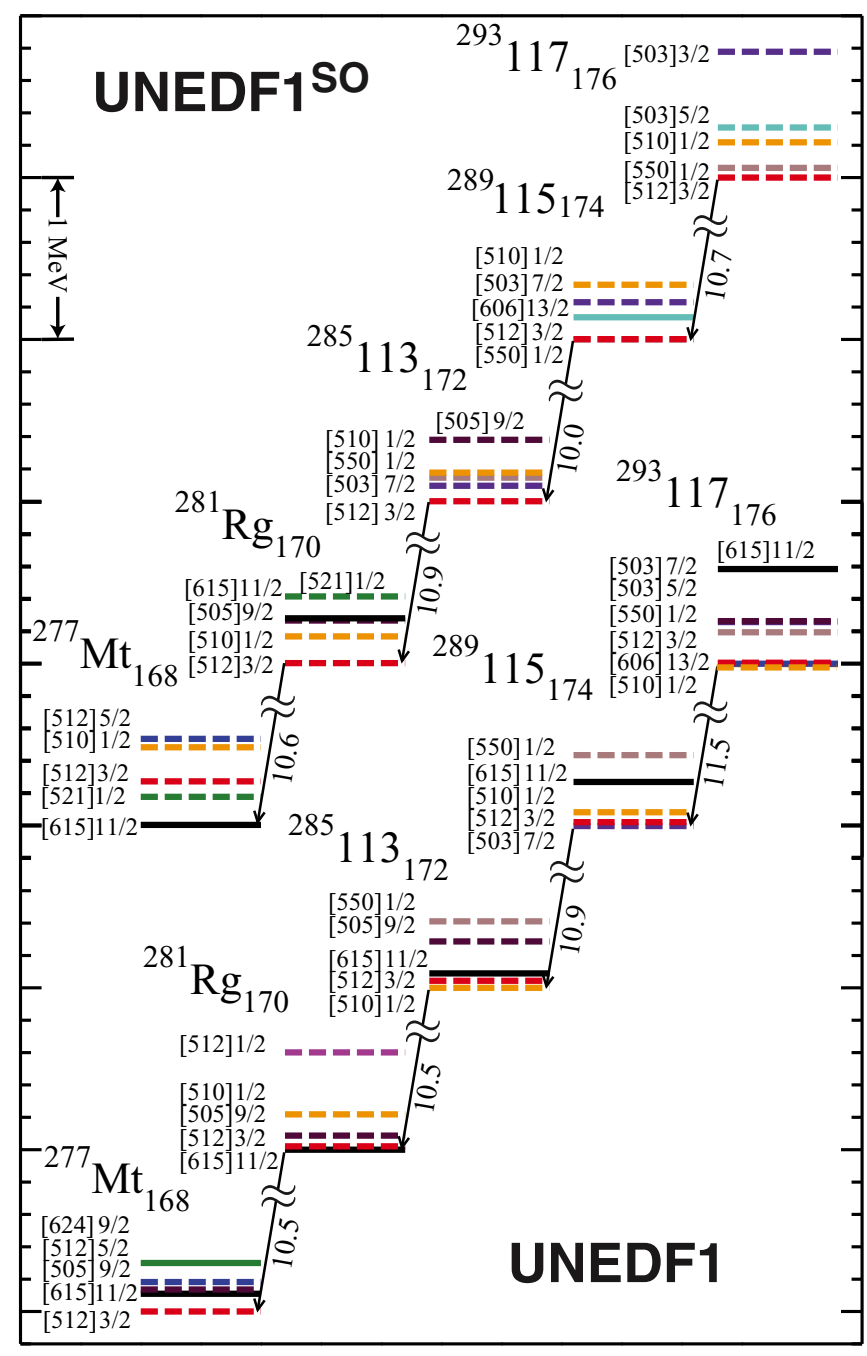

FIG. 6. (Color online) Similar as in Fig. 5 but for the $\alpha$-decay chain ${ }^{293} 117 \rightarrow \cdots \rightarrow{ }^{277} \mathrm{Mt}$.

1-q.p. neutron structure corresponds to the [716]13/2 intruder and $N_{\text {osc }}=6[611] 3 / 2,[613] 5 / 2,[611] 1 / 2$, and [604]9/2 Nilsson orbits. The most significant difference between the two SEDF models is the appearance of the [505]9/2 1-q.p. proton excitation low in energy in UNEDF1. According to the MO model of Fig. 2, the lowest 1-q.p proton excitations are the [615]11/2, [521]1/2, and [505]9/2 Nilsson orbits, while the lowest neutron states are [606]11/2, [604]9/2, $[611] 3 / 2$, [613]5/2, and [716]13/2. It is interesting to note that the structure of 1-q.p. proton states predicted for ${ }^{275} \mathrm{Mt}$ in Ref. [18] falls between predictions of UNEDF1 and UNEDF1 ${ }^{\text {SO }}$. In addition, within the Woods-Saxon model of Ref. [41], the proton states [615]11/2 and [505]9/2 are the two lowest orbitals for Mt over a large range of deformations.

As noted in Ref. [9], there is a very limited choice of q.p. configurations that could generate the observed $E 1$ transitions in ${ }^{276} \mathrm{Mt}$. If one insists on a strict conservation of the $\Omega$ quantum number for protons and neutrons, no low-energy $E 1$ transitions are predicted by $\mathrm{UNEDF}^{\mathrm{SO}}$. Formally, one can construct states that can be connected by an $\Delta \Omega=0, \pm 1$, parity-changing operator, e.g., $\{\pi[615] 11 / 2 \otimes \nu[613] 5 / 2\}_{3^{+}}$
TABLE V. Similar as in Table I but for one-quasi-particle excitations in the odd- $A$ neighbors of ${ }_{109}^{276} \mathrm{Mt}_{167}$ predicted with UNEDF ${ }^{\text {SO }}$ and UNEDF1.

\begin{tabular}{|c|c|c|c|c|}
\hline Nucleus & Config. & $E_{x}(\mathrm{MeV})$ & $Q_{20}$ (b) & $\beta_{2}$ \\
\hline & & UNEDF1 ${ }^{\text {SO }}$ & & \\
\hline \multirow[t]{5}{*}{${ }_{109}^{275} \mathrm{Mt}_{166}$} & {$[615] 11 / 2$} & 0 & 29.7 & 0.23 \\
\hline & {$[512] 3 / 2$} & 0.243 & 29.5 & 0.23 \\
\hline & {$[521] 1 / 2$} & 0.402 & 29.2 & 0.22 \\
\hline & {$[512] 5 / 2$} & 0.500 & 29.7 & 0.23 \\
\hline & {$[510] 1 / 2$} & 0.512 & 29.7 & 0.23 \\
\hline \multirow[t]{5}{*}{${ }_{109}^{277} \mathrm{Mt}_{168}$} & {$[615] 11 / 2$} & 0 & 28.3 & 0.23 \\
\hline & {$[521] 1 / 2$} & 0.174 & 27.4 & 0.21 \\
\hline & {$[512] 3 / 2$} & 0.269 & 28.3 & 0.23 \\
\hline & {$[510] 1 / 2$} & 0.480 & 28.3 & 0.23 \\
\hline & {$[512] 5 / 2$} & 0.532 & 28.3 & 0.23 \\
\hline \multirow{5}{*}{${ }_{108}^{275} \mathrm{Hs}_{167}$} & {$[613] 5 / 2$} & 0 & 28.9 & 0.22 \\
\hline & {$[611] 3 / 2$} & 0.016 & 28.9 & 0.22 \\
\hline & {$[611] 1 / 2$} & 0.117 & 28.9 & 0.22 \\
\hline & {$[604] 9 / 2$} & 0.235 & 28.2 & 0.22 \\
\hline & {$[716] 13 / 2$} & 0.479 & 29.4 & 0.23 \\
\hline \multirow{6}{*}{${ }_{110}^{277} \mathrm{Ds}_{167}$} & {$[611] 3 / 2$} & 0 & 28.6 & 0.22 \\
\hline & {$[613] 5 / 2$} & 0.046 & 28.9 & 0.22 \\
\hline & {$[611] 1 / 2$} & 0.107 & 28.7 & 0.22 \\
\hline & {$[604] 9 / 2$} & 0.335 & 28.6 & 0.22 \\
\hline & {$[716] 13 / 2$} & 0.564 & 29.2 & 0.22 \\
\hline & & UNEDF1 & & \\
\hline \multirow[t]{5}{*}{${ }_{109}^{275} \mathrm{Mt}_{166}$} & {$[512] 3 / 2$} & 0 & 30.0 & 0.23 \\
\hline & {$[615] 11 / 2$} & 0.159 & 29.6 & 0.23 \\
\hline & {$[505] 9 / 2$} & 0.167 & 29.0 & 0.22 \\
\hline & {$[510] 1 / 2$} & 0.173 & 30.1 & 0.23 \\
\hline & {$[624] 9 / 2$} & 0.318 & 29.9 & 0.23 \\
\hline \multirow{5}{*}{${ }_{109}^{277} \mathrm{Mt}_{168}$} & {$[512] 3 / 2$} & 0 & 28.6 & 0.22 \\
\hline & {$[615] 11 / 2$} & 0.131 & 28.2 & 0.21 \\
\hline & {$[505] 9 / 2$} & 0.136 & 27.5 & 0.21 \\
\hline & {$[512] 5 / 2$} & 0.182 & 28.6 & 0.22 \\
\hline & {$[624] 9 / 2$} & 0.300 & 28.5 & 0.21 \\
\hline \multirow[t]{5}{*}{${ }_{108}^{275} \mathrm{Hs}_{167}$} & {$[613] 5 / 2$} & 0 & 29.4 & 0.22 \\
\hline & {$[611] 3 / 2$} & 0.067 & 29.4 & 0.22 \\
\hline & {$[611] 1 / 2$} & 0.104 & 29.5 & 0.22 \\
\hline & {$[716] 13 / 2$} & 0.173 & 29.7 & 0.23 \\
\hline & {$[604] 9 / 2$} & 0.242 & 29.1 & 0.22 \\
\hline \multirow[t]{5}{*}{${ }_{110}^{277} \mathrm{Ds}_{167}$} & {$[611] 3 / 2$} & 0 & 28.9 & 0.22 \\
\hline & {$[613] 5 / 2$} & 0.035 & 29.0 & 0.22 \\
\hline & {$[611] 1 / 2$} & 0.090 & 29.0 & 0.22 \\
\hline & {$[716] 13 / 2$} & 0.157 & 29.2 & 0.22 \\
\hline & {$[604] 9 / 2$} & 0.227 & 28.7 & 0.22 \\
\hline
\end{tabular}

and $\{\pi[521] 1 / 2 \otimes \nu[613] 5 / 2\}_{2^{-}, 3^{-}}$, but a significant Coriolis coupling would be required to produce a measurable $E 1$ rate. The situation is fairly straightforward with UNEDF1. Here, the stretched $E 1$ transition $\pi[505] 9 / 2 \rightarrow \pi[615] 11 / 2$ can explain the data, with the neutron spectator orbital being [611]3/2 or $[613] 5 / 2$ or $[611] 1 / 2$. The NS approach predicts two scenarios: the proton $\pi[615] 11 / 2 \rightarrow \pi[505] 9 / 2$ transition as in UNEDF1 and the neutron $v[716] 13 / 2 \rightarrow v[606] 11 / 2$ transition. According to 2-q.p.-plus-rotor calculations shown in Fig. 7 both scenarios are equally likely. It is interesting to note that the splitting between the $I=1$ and $I=2$ members 


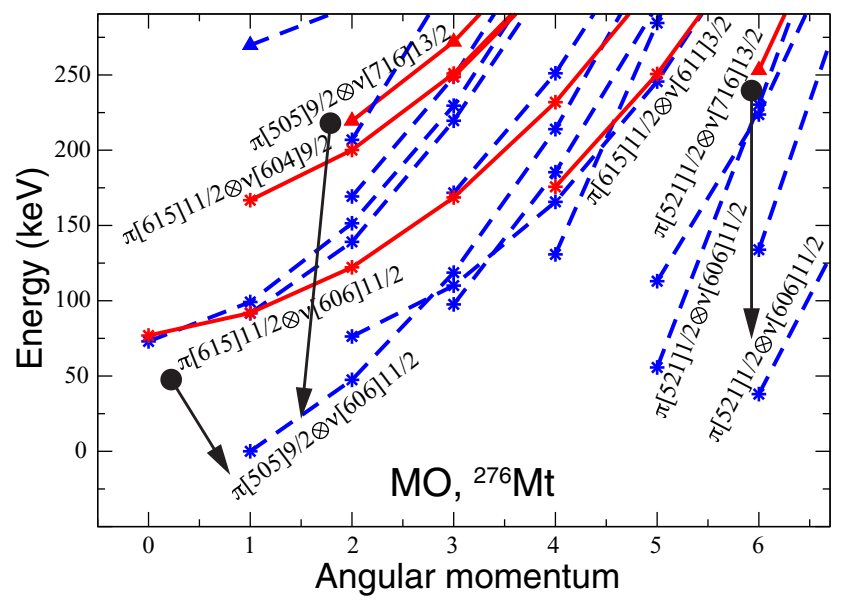

FIG. 7. (Color online) Results of 2-q.p.-plus-rotor NS calculations for ${ }^{276} \mathrm{Mt}$. States connected with lines have the same dominating single-particle configurations; they can be interpreted as rotational bands built on 2-q.p. bandheads indicated. Solid (dashed) lines mark bands with positive (negative) parity. Positive (negative) parity neutron configurations are indicated by asterisks (triangles). The candidates for stretched $\Delta \Omega=0, \pm 1 E 1$ transitions are marked by thick arrows.

of the lowest $K^{\pi}=1^{-}(\pi[505] 9 / 2 \otimes \nu[606] 11 / 2)$ band is only $43 \mathrm{keV}$; see Fig. 7 . This is particularly close to the energy difference $\approx 47 \mathrm{keV}$ between the states suggested experimentally [9].

To analyze the case of ${ }^{272} \mathrm{Bh}$, we have calculated the 1-q.p. spectra of ${ }^{271,273} \mathrm{Bh},{ }^{273} \mathrm{Hs}$, and ${ }^{271} \mathrm{Sg}$; see Table VI. The resulting level scheme is very complex. Indeed, as seen in Fig. 8, 2-q.p.-plus-rotor calculations predict quite a few candidates for low-energy $E 2$ and $M 1$ transitions, and this is consistent with experiment [9]. The results displayed in Figs. 7 and 8 clearly demonstrate that a small change in ordering of Nilsson orbits can influence the decay scenarios.

The calculated $Q_{\alpha}$ values depend, of course, on the structure of parent and daughter states [20] (see Figs. 5 and 6). The agreement with the measured values for the heaviest elements

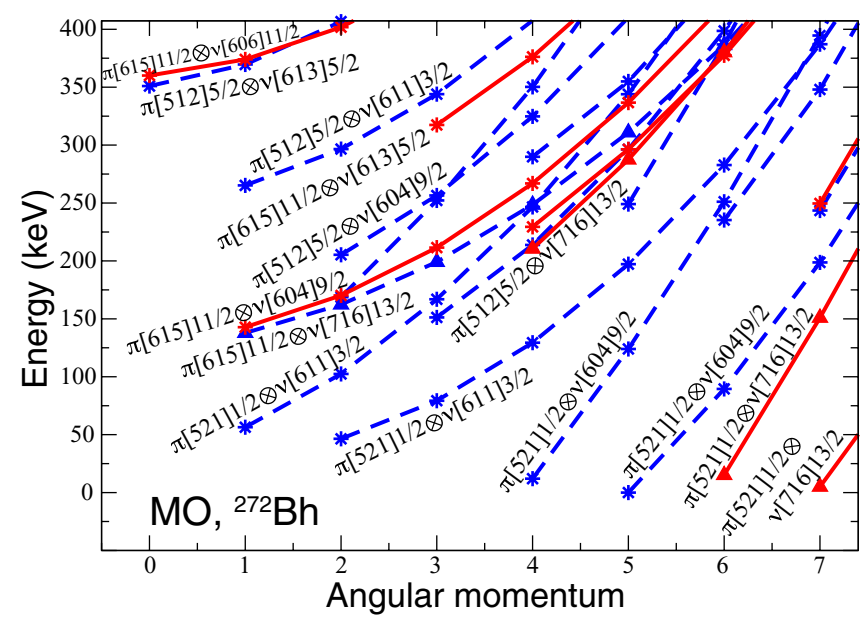

FIG. 8. (Color online) Similar as in Fig. 7 but for ${ }^{272} \mathrm{Bh}$.
TABLE VI. Similar as in Table $\mathrm{V}$ but for one-quasiparticle excitations in the odd- $A$ neighbors of ${ }_{107}^{272} \mathrm{Bh}_{165}$ predicted with $\mathrm{UNEDF}^{\mathrm{SO}}$ and UNEDF1.

\begin{tabular}{|c|c|c|c|c|}
\hline Nucleus & Config. & $E_{x}(\mathrm{MeV})$ & $Q_{20}(\mathrm{~b})$ & $\beta_{2}$ \\
\hline & & $\mathrm{UNEDF}_{1}$ SO & & \\
\hline \multirow[t]{5}{*}{${ }_{107}^{271} \mathrm{Bh}_{164}$} & {$[512] 5 / 2$} & 0 & 30.8 & 0.24 \\
\hline & {$[521] 1 / 2$} & 0.076 & 30.3 & 0.23 \\
\hline & {$[615] 11 / 2$} & 0.244 & 30.4 & 0.23 \\
\hline & {$[624] 9 / 2$} & 0.415 & 30.8 & 0.24 \\
\hline & {$[514] 7 / 2$} & 0.682 & 31.1 & 0.24 \\
\hline \multirow[t]{6}{*}{${ }_{107}^{273} \mathrm{Bh}_{166}$} & {$[615] 11 / 2$} & 0 & 29.3 & 0.23 \\
\hline & {$[512] 5 / 2$} & 0.002 & 29.6 & 0.23 \\
\hline & {$[521] 1 / 2$} & 0.072 & 29.5 & 0.23 \\
\hline & {$[624] 9 / 2$} & 0.243 & 29.6 & 0.23 \\
\hline & {$[512] 3 / 2$} & 0.574 & 29.4 & 0.23 \\
\hline & {$[514] 7 / 2$} & 0.692 & 29.8 & 0.23 \\
\hline \multirow[t]{5}{*}{${ }_{106}^{271} \mathrm{Sg}_{165}$} & {$[611] 3 / 2$} & 0 & 30.1 & 0.23 \\
\hline & {$[716] 13 / 2$} & 0.156 & 30.2 & 0.23 \\
\hline & {$[613] 5 / 2$} & 0.223 & 30.1 & 0.23 \\
\hline & {$[611] 1 / 2$} & 0.311 & 30.1 & 0.23 \\
\hline & {$[604] 9 / 2$} & 0.429 & 29.2 & 0.23 \\
\hline \multirow[t]{6}{*}{${ }_{108}^{273} \mathrm{Hs}_{165}$} & {$[611] 3 / 2$} & 0 & 30.1 & 0.23 \\
\hline & {$[613] 5 / 2$} & 0.118 & 30.0 & 0.23 \\
\hline & {$[716] 13 / 2$} & 0.142 & 30.3 & 0.23 \\
\hline & {$[611] 1 / 2$} & 0.314 & 30.1 & 0.23 \\
\hline & {$[604] 9 / 2$} & 0.518 & 29.2 & 0.22 \\
\hline & & UNEDF1 & & \\
\hline \multirow[t]{5}{*}{${ }_{107}^{271} \mathrm{Bh}_{164}$} & {$[512] 5 / 2$} & 0 & 31.6 & 0.24 \\
\hline & {$[624] 9 / 2$} & 0.028 & 31.5 & 0.24 \\
\hline & {$[512] 3 / 2$} & 0.338 & 31.3 & 0.24 \\
\hline & {$[521] 1 / 2$} & 0.554 & 30.9 & 0.24 \\
\hline & {$[505] 9 / 2$} & 0.714 & 30.0 & 0.23 \\
\hline \multirow{5}{*}{${ }_{107}^{273} \mathrm{Bh}_{166}$} & {$[624] 9 / 2$} & 0 & 30.7 & 0.23 \\
\hline & {$[512] 5 / 2$} & 0.076 & 30.7 & 0.23 \\
\hline & {$[512] 3 / 2$} & 0.306 & 30.6 & 0.23 \\
\hline & {$[521] 1 / 2$} & 0.377 & 29.6 & 0.23 \\
\hline & {$[505] 9 / 2$} & 0.599 & 28.7 & 0.22 \\
\hline \multirow[t]{5}{*}{${ }_{106}^{271} \mathrm{Sg}_{165}$} & {$[611] 3 / 2$} & 0 & 31.1 & 0.24 \\
\hline & {$[613] 5 / 2$} & 0.007 & 30.7 & 0.24 \\
\hline & {$[716] 13 / 2$} & 0.11 & 31.1 & 0.24 \\
\hline & {$[611] 1 / 2$} & 0.252 & 31.0 & 0.24 \\
\hline & {$[604] 9 / 2$} & 0.635 & 33.2 & 0.25 \\
\hline \multirow[t]{5}{*}{${ }_{108}^{273} \mathrm{Hs}_{165}$} & {$[613] 5 / 2$} & 0 & 30.7 & 0.24 \\
\hline & {$[611] 3 / 2$} & 0.048 & 30.8 & 0.24 \\
\hline & {$[716] 13 / 2$} & 0.119 & 30.8 & 0.24 \\
\hline & {$[611] 1 / 2$} & 0.300 & 30.8 & 0.24 \\
\hline & {$[604] 9 / 2$} & 0.508 & 30.4 & 0.23 \\
\hline
\end{tabular}

is reasonable, usually better than $1 \mathrm{MeV}$. This is comparable with other calculations [14,42-44].

\section{CONCLUSIONS}

In summary, we studied shell structure of superheavy nuclei within the self-consistent SHFB approach and macroscopicmicroscopic NS model. Detailed predictions have been made for the quasiproton and quasineutron structures of nuclei belonging to the $\alpha$-decay chains of ${ }^{287} 115,{ }^{287} \mathrm{Lv},{ }^{289} \mathrm{Lv}$, and 
${ }^{293} 117$. The UNEDF1 and UNEDF1 ${ }^{\text {SO }}$ SEDF models differ in the strength of the spin-orbit term, and this impacts detailed predictions for the deformed nuclei around $Z=110$ and $N=$ 168. The recent observation of low-energy $E 1$ transitions in ${ }^{276} \mathrm{Mt}$ [9] provides a stringent constraint on theoretical models. Indeed, the recently proposed UNEDF ${ }^{\mathrm{SO}}$ parametrization that performs well in the transfermium region does not offer a simple explanation of the $E 1$ data, whereas the global UNEDF1 parametrization explains the data in terms of the proton $\pi[505] 9 / 2 \rightarrow \pi[615] 11 / 2$ transition. The MO models suggests two competing scenarios: a proton transition similar to that of UNEDF1 and an alternative neutron $v[716] 13 / 2 \rightarrow$ $v[606] 11 / 2 E 1$ transition. To confirm or disprove these scenarios, theory strongly recommends a search for $E 1$ transitions in neighboring odd- $A$ systems ${ }^{275,277} \mathrm{Mt},{ }^{275} \mathrm{Hs}$, and ${ }^{277}$ Ds. Experimentally, this calls for high-resolution $\alpha$ photon coincidence spectroscopy of decay chains starting from ${ }^{293} 117,{ }^{287,289} 115$, or ${ }^{285,287} \mathrm{Fl}$, respectively. However, the observation of these systems is hampered either by relatively low production cross sections or large spontaneous fission branches on the way to the nuclei of structural interest [1-9].
A solution to this spectroscopic puzzle may significantly contribute to our understanding of shell structure in superheavy nuclei, and the strength of the spin-orbit splitting in particular.

\section{ACKNOWLEDGMENTS}

Discussions with S. Åberg are gratefully acknowledged. This work was supported by the U.S. Department of Energy (DOE) under Contracts No. DE-FG02-96ER40963 (University of Tennessee), No. DE-SC0008499 (NUCLEI SciDAC Collaboration), and No. DE-NA0001820 (the Stewardship Science Academic Alliances program); by the Academy of Finland and University of Jyväskylä within the FIDIPRO program; by the Polish National Science Center under Contract No. 2012/07/B/ST2/03907; and by the Swedish Research Council. A grant of computer time was provided by the National Institute for Computational Sciences (NICS) and the Innovative and Novel Computational Impact on Theory and Experiment (INCITE) program using resources of the OLCF facility.
[1] Y. T. Oganessian, J. Phys. G 34, R165 (2007).

[2] Y. T. Oganessian, Radiochim. Acta 99, 429 (2011).

[3] Y. T. Oganessian et al., Phys. Rev. Lett. 108, 022502 (2012).

[4] Y. T. Oganessian et al., Phys. Rev. Lett. 109, 162501 (2012).

[5] Y. T. Oganessian et al., Phys. Rev. C 87, 014302 (2013).

[6] C. E. Düllmann et al., Phys. Rev. Lett. 104, 252701 (2010).

[7] J. M. Gates et al., Phys. Rev. C 83, 054618 (2011).

[8] S. Hofmann et al., Eur. Phys. J. A 48, 62 (2012).

[9] D. Rudolph et al., Phys. Rev. Lett. 111, 112502 (2013).

[10] S. Ćwiok, J. Dobaczewski, P.-H. Heenen, P. Magierski, and W. Nazarewicz, Nucl. Phys. A 611, 211 (1996).

[11] A. T. Kruppa, M. Bender, W. Nazarewicz, P.-G. Reinhard, T. Vertse, and S. Ćwiok, Phys. Rev. C 61, 034313 (2000).

[12] M. Bender, W. Nazarewicz, and P.-G. Reinhard, Phys. Lett. B 515, 42 (2001).

[13] S. Ćwiok, P.-H. Heenen, and W. Nazarewicz, Nature (London) 433, 705 (2005).

[14] M. Bender and P.-H. Heenen, J. Phys.: Conf. Ser. 420, 012002 (2013).

[15] Y. Shi, J. Dobaczewski, and P. T. Greenlees, Phys. Rev. C 89, 034309 (2014).

[16] D. Rudolph et al., Acta Phys. Pol. B 45, 263 (2014).

[17] S. Ćwiok, S. Hofmann, and W. Nazarewicz, Nucl. Phys. A 573, 356 (1994).

[18] A. Parkhomenko and A. Sobiczewski, Acta Phys. Pol. B 35, 2447 (2004).

[19] A. Parkhomenko and A. Sobiczewski, Acta Phys. Pol. B 36, 3115 (2005).

[20] S. Ćwiok, W. Nazarewicz, and P. H. Heenen, Phys. Rev. Lett. 83, 1108 (1999).

[21] M. Bender, Phys. Rev. C 61, 031302 (2000).

[22] M. Bender, P.-H. Heenen, and P.-G. Reinhard, Rev. Mod. Phys. 75, 121 (2003).

[23] J. Erler, N. Birge, M. Kortelainen, W. Nazarewicz, E. Olsen, A. Perhac, and M. Stoitsov, Nature (London) 486, 509 (2012).
[24] M. Kortelainen, J. McDonnell, W. Nazarewicz, P.-G. Reinhard, J. Sarich, N. Schunck, M. V. Stoitsov, and S. M. Wild, Phys. Rev. C 85, 024304 (2012).

[25] N. Schunck, J. Dobaczewski, J. McDonnell, W. Satuła, J. Sheikh, A. Staszczak, M. Stoitsov, and P. Toivanen, Comput. Phys. Commun. 183, 166 (2012).

[26] N. Schunck, J. Dobaczewski, J. McDonnell, J. Moré, W. Nazarewicz, J. Sarich, and M. V. Stoitsov, Phys. Rev. C 81, 024316 (2010).

[27] B. G. Carlsson and I. Ragnarsson, Phys. Rev. C 74, 011302 (2006).

[28] S. G.Nilsson, C. F.Tsang, A. Sobiczewski, Z. Szymański, S. Wycech, C. Gustafson, I.-L. Lamm, P. Möller, and B. Nilsson, Nucl. Phys. A 131, 1 (1969).

[29] P. Rozmej, K. Boning, and A. Sobiczewski, in Proceedings of the XXIV International Winter Meeting on Nuclear Physics, Bormio, Italy, edited by I. Iori (Ric. Sci. Educ. Permanente, Milano, 1986), p. 567.

[30] C. Herrmann et al., Nucl. Phys. A 493, 83 (1989).

[31] A. B. Hayes et al., Phys. Rev. C 82, 044319 (2010).

[32] I. Ragnarsson and P. B. Semmes, Hyperfine Interact. 43, 423 (1988)

[33] L. Grodzins, Phys. Lett. 2, 88 (1962).

[34] W. Nazarewicz and I. Ragnarsson, in Handbook of Nuclear Properties, edited by D. N. Poenaru and W. Greiner (Clarendon Press, Oxford, 1996), p. 80.

[35] C. Gustafson, I. L. Lamm, B. Nilsson, and S. G. Nilsson, Ark. Fys. 36, 613 (1967).

[36] U. Mosel and W. Greiner, Z. Phys. 222, 261 (1969).

[37] M. Bolsterli, E. O. Fiset, J. R. Nix, and J. L. Norton, Phys. Rev. C 5, 1050 (1972).

[38] J. R. Nix, Ann. Rev. Nucl. Sci. 22, 65 (1972).

[39] P. Möller and J. Nix, J. Phys. G 20, 1681 (1994).

[40] M. Bender, K. Rutz, P.-G. Reinhard, J. A. Maruhn, and W. Greiner, Phys. Rev. C 60, 034304 (1999). 
[41] R. R. Chasman, I. Ahmad, A. M. Friedman, and J. R. Erskine, Rev. Mod. Phys. 49, 833 (1977).

[42] V. Prassa, T. Nikšić, G. A. Lalazissis, and D. Vretenar, Phys. Rev. C 86, 024317 (2012).
[43] M. Warda and J. L. Egido, Phys. Rev. C 86, 014322 (2012).

[44] A. Staszczak, A. Baran, and W. Nazarewicz, Phys. Rev. C 87, 024320 (2013). 